\title{
ERRATUM
}

\author{
Sebastian Burciu
}

\section{Erratum to: On the Representation Theory of Normal Hopf Subalgebras}

Published online: 19 October 2011

(C) King Fahd University of Petroleum and Minerals 2011

\section{Erratum to: Arab J Sci Eng \\ DOI 10.1007/s13369-011-0094-0}

During separation of the numbering of equations/formulas from that of Theorems/Propositions at the proof correction stage, errors were introduced by the typesetter. The correct numbering should be as follows:

Remark $4.3=$ Remark 4.1

Theorem $4.5=$ Theorem 4.2

Corollary $4.6=$ Corollary 4.3

Definition $4.7=$ Definition 4.4

Definition $4.8=$ Definition 4.5

Theorem $4.9=$ Theorem 4.6

The online version of the original article can be found under doi:10.1007/s13369-011-0094-0.

S. Burciu

Institute of Mathematics "Simion Stoilow" of the Romanian Academy, P.O. Box 1-764, 014700 Bucharest, Romania

S. Burciu $(\square)$

Faculty of Mathematics and Computer Science, University of Bucharest, 14 Academiei Street, Bucharest, Romania

E-mail: sebastian.burciu@imar.ro 